\title{
Characterization of bone marrow derived mesenchymal stem cells in suspension
}

Kentaro Akiyama ${ }^{1,2+}$, Yong-Ouk You ${ }^{1+}$, Takayoshi Yamaza ${ }^{1,3}$, Chider Chen ${ }^{1}$, Liang Tang ${ }^{4}$, Yan Jin ${ }^{4}$, Xiao-Dong Chen ${ }^{5}$, Stan Gronthos ${ }^{6}$ and Songtao Shi ${ }^{*}$

\begin{abstract}
Introduction: Bone marrow mesenchymal stem cells (BMMSCs) are a heterogeneous population of postnatal precursor cells with the capacity of adhering to culture dishes generating colony-forming unit-fibroblasts (CFU-F). Here we identify a new subset of BMMSCs that fail to adhere to plastic culture dishes and remain in culture suspension (S-BMMSCs).

Methods: To catch S-BMMSCs, we used BMMSCs-produced extracellular cell matrix (ECM)-coated dishes. Isolated S-BMMSCs were analyzed by in vitro stem cell analysis approaches, including flow cytometry, inductive multiple differentiation, western blot and in vivo implantation to assess the bone regeneration ability of S-BMMSCS. Furthermore, we performed systemic S-BMMSCs transplantation to treat systemic lupus erythematosus (SLE)-like MRL/Ipr mice.

Results: S-BMMSCs are capable of adhering to ECM-coated dishes and showing mesenchymal stem cell characteristics with distinction from hematopoietic cells as evidenced by co-expression of CD73 or Oct-4 with CD34, forming a single colony cluster on ECM, and failure to differentiate into hematopoietic cell lineage. Moreover, we found that culture-expanded S-BMMSCs exhibited significantly increased immunomodulatory capacities in vitro and an efficacious treatment for SLE-like MRL/Ipr mice by rebalancing regulatory T cells (Tregs) and Thelper 17 cells (Th17) through high NO production.
\end{abstract}

Conclusions: These data suggest that it is feasible to improve immunotherapy by identifying a new subset BMMSCS.

\section{Introduction}

Bone marrow mesenchymal stem cells (BMMSCs) are hierarchical postnatal stem/progenitor cells capable of self-renewing and differentiating into osteoblasts, chondrocytes, adipocytes, and neural cells [1,2]. BMMSCs express a unique surface molecule profile, including expression of STRO-1, CD29, CD73, CD90, CD105, CD146, Octamer-4 (Oct4), and stage-specific embryonic antigen-4 (SSEA4) $[3,4]$. It is generally believed that BMMSCs are negative for hematopoietic cell markers such as CD14 and CD34 [5-13]. BMMSCs have been widely used for tissue engineering [14-16]. Recently, a growing body of evidence has indicated that BMMSCs

\footnotetext{
*Correspondence: songtaos@usc.edu

† Contributed equally

'Center for Craniofacial Molecular Biology, University of Southern California, 2250 Alcazar Street, CSA 103, Los Angeles, CA 90033, USA Full list of author information is available at the end of the article
}

produce a variety of cytokines and display profound immunomodulatory properties [17-19], perhaps by inhibiting the proliferation and function of several major immune cells, such as natural killer cells, dendritic cells, and $T$ and $B$ lymphocytes [17-20]. These unique properties make BMMSCs of great interest for clinical applications in the treatment of different immune disorders $[17,21-24]$.

BMMSCs are thought to be derived from the bone marrow stromal compartment, initially appearing as adherent, single colony clusters (colony-forming unitfibroblasts [CFU-F]), and subsequently proliferating on culture dishes [25]. To date, the CFU-F assay has been considered one of the gold standards for determining the incidence of clonogenic BMMSC $[26,27]$. Since BMMSC are a heterogeneous population of stem cells, it is critical to identify whether BMMSC contain unique cell subsets with distinctive functions, analogous to the
C Biomed Central 
hematopoietic stem/progenitor cell system. In this study, we identified a subset of mouse BMMSCs in culture suspension and determined their immunomodulatory characteristics.

\section{Materials and methods \\ Animals}

Female C3H/HeJ, C57BL/6J, and C3MRL-Fas ${ }^{\mathrm{lpr}} / \mathrm{J}$ mice were purchased from Jackson Laboratory (Bar Harbor, ME, USA). Female immunocompromised mice (Beige nude/nude XIDIII) were purchased from Harlan (Indianapolis, IN, USA). All animal experiments were performed under the institutionally approved protocols for the use of animal research (USC \#10874 and 10941).

\section{Antibodies}

Anti Oct4, SSEA4, Runx2, OCN, active $\beta$ catenin and $\beta$ catenin were purchased from Millipore (Billerica, MA, USA). Anti alkaline phosphatase (ALP) antibody was purchased from Abcam (Cambridge, MA, USA). Anti Sca-1-PE, CD34-PE, CD34-FITC, CD45-PE, CD73-PE, CD4-PerCP, CD8-FITC, CD25-APC, CD3e and CD28 antibodies were purchased from BD Bioscience (San Jose, CA, USA). Anti Foxp3-PE, IL17-PE, and IFN $\gamma$-APC antibodies were purchased from eBioscience (San Diego, CA, USA). Unconjugated anti CD34, CD73, and CD105, NOS2 were purchased from Santa Cruz Biosciences (Santa Cruz, CA, USA). Anti $\beta$ actin antibody was purchased from Sigma (St. Louis, MO, USA).

\section{Isolation of mouse bone marrow mesenchymal stem cells (BMMSCs)}

The single suspension of bone marrow derived all nucleated cells (ANCs) from femurs and tibias were seeded at a density of $15 \times 10^{6}$ into $100 \mathrm{~mm}$ culture dishes (Corning, NY, USA) at $37^{\circ} \mathrm{C}$ and $5 \% \mathrm{CO} 2$. Nonadherent cells were removed after two days and attached cells were maintained for 16 days in alpha minimum essential medium ( $\alpha$-MEM, Invitrogen, Grand Island, NY, USA) supplemented with $20 \%$ fetal bovine serum (FBS, Equitech-bio, Kerrville, TX, USA), 2 mM L-glutamine, $55 \mu \mathrm{M}$ 2-mercaptoethanol, $100 \mathrm{U} / \mathrm{ml}$ penicillin, and $100 \mu \mathrm{g} / \mathrm{ml}$ streptomycin (Invitrogen). Colony-forming attached cells were passed once for further experimental use.

\section{Preparation of Extracellular Matrix (ECM) coated dishes}

ECM coated dishes were prepared as described previously [28]. Briefly, 100\% confluence of BMMSCs was cultured in medium with $100 \mathrm{nM}$ L-ascorbic acid phosphate (Wako Pure Chemical, Richmond, VA, USA). After two weeks, cultures were washed with PBS and incubated with $0.005 \%$ Triton X-100 (Sigma) for 15 minutes at room temperature to remove cells. The ECM was treated with DNase I (100 units/ml; Sigma) for 1 hour at $37^{\circ} \mathrm{C}$. The ECM was washed with PBS three times and stored in $2 \mathrm{ml}$ of PBS containing $100 \mathrm{U} / \mathrm{ml}$ penicillin, $100 \mu \mathrm{g} / \mathrm{ml}$ streptomycin and $0.25 \mu \mathrm{g} / \mathrm{ml}$ fungizone (Invitrogen) at $4^{\circ} \mathrm{C}$.

Isolation of BMMSCs in culture suspension (S-BMMSCs)

Bone marrow-derived ANCs $\left(15 \times 10^{6}\right)$ were seeded into $100 \mathrm{~mm}$ culture dishes and cultured for two days. The culture supernatant with floating cells was collected and centrifuged to obtain putative non-attached BMMSCs. The cells were re-seeded at indicated numbers on ECMcoated dishes. After 2 days, the floating cells in the cultures were removed with PBS and the attached cells on ECM were maintained for an additional 14 days. Colony-forming attached cells were passed once and subcultured on regular plastic culture dishes for further experiments. For some stem cell characterization analyses, we collected SSEA4 positive S-BMMSCs using the MACS magnetic separation system (Milteny Biotech, Auburn, CA, USA) and expanded in the cultures.

\section{Colony forming unit-fibroblastic (CFU-F) assay}

One million cells of ANCs from bone marrow were seeded on a T-25 cell culture flask (Nunc, Rochester, NY, USA). After 16 days, the cultures were washed with PBS and stained with $1 \%$ toluidine blue solution in $2 \%$ paraformaldehyde (PFA). A cell cluster that had more than 50 cells was counted as a colony under microscopy. The colony number was counted in five independent samples per each experimental group.

\section{Cell proliferation assay}

The proliferation of BMMSCs and S-BMMSCs was performed using the bromodeoxyuridine (BrdU) incorporation assay. Each cell population $\left(1 \times 10^{4}\right.$ cells/well $)$ was seeded on two-well chamber slides (Nunc) and cultured for two to three days. The cultures were incubated with BrdU solution (1:100) (Invitrogen) for 20 hours, and stained with a BrdU staining kit (Invitrogen). BrdU-positive and total cell numbers were counted in ten images per subject. The BrdU assay was repeated in five independent samples for each experimental group.

\section{Population doubling assay}

A total of $0.5 \times 10^{6}$ cells of BMMSCs and S-BMMSCs was seeded on $60 \mathrm{~mm}$ culture dishes at the first passage. Upon reaching confluence, the cells were passaged at the same cell density. The population doubling was calculated at every passage according to the equation: $\log _{2}$ (number of harvested cells/number of seeded cells). The finite population doublings were determined by cumulative addition of total numbers generated from each passage until the cells ceased dividing. 
Flow cytometric analysis of mesenchymal stem cell surface molecules

BMMSCs or S-BMMSCs $\left(0.2 \times 10^{6}\right.$ cells $)$ were incubated with $1 \mu \mathrm{g}$ of R-Phycoerythrin (PE). (PE)-conjugated antibodies or isotype-matched control immunoglobulin Gs (IgGs) (Southern Biotech, Birmingham, AL, USA) at $4^{\circ} \mathrm{C}$ for 45 minutes. Samples were analyzed by a fluorescenceactivated cell sorting (FACS) ${ }^{\text {Calibur }}$ flow cytometer (BD Bioscience). For dual color analysis, the cells were treated with PE-conjugated and fluorescein isothiocyanate (FITC)-conjugated antibodies or isotype-matched control IgGs $\left(1 \mu \mathrm{g}\right.$ each). The cells were analyzed on FACS ${ }^{\text {Calibur }}$ (BD Bioscience).

\section{Immunofluorescent microscopy}

The cells subcultured on eight-well chamber slides (Nunc) $\left(2 \times 10^{3} /\right.$ well $)$ were fixed with $4 \%$ PFA. The samples were incubated with the specific or isotypematched mouse antibodies (1:200) overnight at $4^{\circ} \mathrm{C}$, and treated with Rhodamine-conjugated secondary antibodies (1:400, Jackson ImmunoResearch, West Grove, PA, USA; Southern Biotechnology, Birmingham, AL, USA). Finally, chamber slides were mounted using Vectashield mounting medium containing 4', 6-diamidino-2-phenylindole (DAPI) (Vector Laboratories, Burlingame, CA, USA).

\section{In vivo bone formation assay}

A total of $4.0 \times 10^{6}$ cells was mixed with hydroxyapatite/tricalcium phosphate (HA/TCP) ceramic powders (40 mg, Zimmer Inc., Warsaw, IN, USA) and subcutaneously transplanted into eight-week-old immunocompromised mice. After eight weeks, the transplants were harvested, fixed in 4\% PFA and then decalcified with 5\% ethylenediaminetetraacetic acid (EDTA; pH 7.4), followed by paraffin embedding. The paraffin sections were stained with $\mathrm{H} \& \mathrm{E}$ and analyzed by an NIH Image-J. The newly-formed mineralized tissue area from five fields was calculated and shown as a percentage to total tissue area.

\section{In vitro osteogenic differentiation assay}

BMMSCs and S-BMMSCs were cultured under osteogenic culture conditions containing $2 \mathrm{mM} \beta$-glycerophosphate (Sigma), $100 \mu \mathrm{M}$ L-ascorbic acid 2-phosphate and $10 \mathrm{nM}$ dexamethasone (Sigma). After induction, the cultures were stained with alizarin red or alkaline phosphatase.

\section{In vitro adipogenic differentiation assay}

For adipogenic induction, $500 \mathrm{nM}$ isobutylmethylxanthine, $60 \mu \mathrm{M}$ indomethacin, $500 \mathrm{nM}$ hydrocortisone, $10 \mu \mathrm{g} / \mathrm{ml}$ insulin (Sigma), $100 \mathrm{nM} \mathrm{L-ascorbic} \mathrm{acid} \mathrm{phos-}$ phate were added to the culture medium. After 10 days, the cultured cells were stained with Oil Red-O and positive cells were quantified by using an NIH Image-J. Total RNA was also isolated from cultures after 10 days induction for further experiments.

\section{In vitro chondrogenic differentiation assay}

For chondrogenic induction, $1 \times 10^{6}$ cell pellets were cultured under chondrogenic medium containing $15 \% \mathrm{FBS}$, $1 \%$ ITS (BD), $100 \mathrm{nM}$ dexamethasone, $2 \mathrm{mM}$ pyruvate (SIGMA), and $10 \mathrm{ng} / \mathrm{ml}$ transforming growth factor beta 1 (TGF $\beta 1$ ) in (D)MEM (Invitrogen) for threeweeks. Cell pellets were harvested at three weeks post induction, fixed overnight with $4 \%$ PFA and then, sections were prepared for staining.

\section{Reverse transcriptase polymerase chain reaction (RT-PCR)} analysis

Extraction of total RNA and RT-PCR were performed according to standard procedures. Primer information is described in Additional materials and methods [see Additional file 1].

\section{Western blotting analysis}

A total of $20 \mu \mathrm{g}$ of protein was used and SDS-PAGE and western blotting were performed according to standard procedures. Detailed procedures are described in Additional materials and methods [see Additional file 1]. $\beta$-actin on the same membrane served as the loading control.

Hematopoietic differentiation of BMMSCs and S-BMMSCs BMMSCs and S-BMMSCs were cultured onto $35 \mathrm{~mm}$ low attach culture dishes $\left(2 \times 10^{4} /\right.$ dish, STEMCELL Technologies, Vancouver, BC, V5Z 1B3, Canada) under hematopoietic differentiation medium (STEMCELL Technologies) with or without erythropoietin (EPO; 3 $\mathrm{U} / \mathrm{mL}$ ) for seven days. Whole bone marrow cells and linage negative bone marrow cells (Linage-cells) were used as positive controls. The results are representative of five independent experiments.

\section{Inhibitor treatment}

S-BMMSCs and BMMSCs were treated with $1 \mathrm{mM} \mathrm{L-}$ NG-monomethyl-arginine (L-NMMA) (Cayman Chemical, Ann Arbor, MI, USA) or 0.2 mM 1400 W (Cayman Chemical) to inhibit total nitric oxide synthase (NOS) or inducible nitric oxide synthase (iNOS), respectively.

\section{Measurement of nitric oxide production}

BMMSCs $\left(0.2 \times 10^{6} /\right.$ well $)$ were cultured on 24 -well plates with or without cytokines (IFN $\gamma, 25 \mathrm{ng} / \mathrm{ml}$; IL- $1 \beta, 5 \mathrm{ng} / \mathrm{ml}$, R\&D Systems, Minneapolis, MN, USA) and chemicals (L-NMMA, $1 \mathrm{mM} ; 1400 \mathrm{~W}, 0.2 \mathrm{mM}$ ) at the indicated concentration and days. The supernatant from each culture was collected and nitric oxide concentration measured using a Total Nitric Oxide and Nitrate/Nitrite Parameter 
Assay kit (R\&D Systems) according to the manufacturer's instruction.

\section{Cell apoptosis and cell survival assay}

The transwell system (Corning) was used for co-culture experiments. A total of $0.2 \times 10^{6}$ of S-BMMSCs or BMMSCs was seeded on each lower chamber. Activated spleen cells $\left(1 \times 10^{6} /\right.$ chamber $)$, which were pre-stimulated with plate-bound anti CD3 $\varepsilon$ antibody $(3 \mu \mathrm{g} / \mathrm{ml})$ and soluble anti CD28 antibody $(2 \mu \mathrm{g} / \mathrm{ml})$ for two days, were loaded in the upper chambers. Both chambers were filled with a complete medium containing (D) MEM (Lonza, CH-4002 Basel, Switzerland) with 10\% heat-inactivated FBS, $50 \mu \mathrm{M}$ 2-mercaptoethanol, $10 \mathrm{mM}$ HEPES, $1 \mathrm{mM}$ sodium pyruvate (Sigma), 1\% non-essential amino acid (Cambrex, East Rutherford, NJ, USA), 2 $\mathrm{mM} \mathrm{L}$-glutamine, $100 \mathrm{U} / \mathrm{ml}$ penicillin and $100 \mathrm{mg} / \mathrm{ml}$ streptomycin. To measure the spleen cells viability, cell counting kit-8 (Dojindo Molecular Technologies, Rockville, MD, USA) was used. For apoptosis of spleen cells analyses, Annexin V-PE apoptosis detection kits I (BD Bioscience) were used and analyzed on FACS ${ }^{\text {Calibur }}$ (BD Bioscience).

\section{In vitro $\mathrm{CD}^{+} \mathrm{CD}^{2} 5^{+} \mathrm{Foxp}^{+}{ }^{+}$Tregs and Th17 induction} $\mathrm{CD} 4{ }^{+} \mathrm{CD} 25^{-} \mathrm{T}$-lymphocytes $\left(1 \times 10^{6} /\right.$ well $)$, collected using a $\mathrm{CD} 4^{+} \mathrm{CD} 25^{+}$Treg isolation kit (Miltenyi Biotec), were pre-stimulated with plate-bound anti $\mathrm{CD} 3 \varepsilon$ antibody $(3 \mu \mathrm{g} / \mathrm{ml})$ and soluble anti CD28 antibody $(2 \mu \mathrm{g} /$ $\mathrm{ml}$ ) for two days. These activated T-lymphocytes were loaded on $0.2 \times 10^{6}$ BMMSCs or S-BMMSCs cultures with recombinant human TFG $\beta 1(2 \mathrm{ng} / \mathrm{ml})$ (R\&D Systems) and recombinant mouse IL2 $(2 \mathrm{ng} / \mathrm{ml})$ (R\&D Systems). For Th17 induction, recombinant human TFG $\beta 1$ $(2 \mathrm{ng} / \mathrm{ml})$ and recombinant mouse IL6 $(50 \mathrm{ng} / \mathrm{ml})$ (Biolegend, San Diego, CA, USA) were added. After three days, cells in suspension were collected and stained with anti CD4-PerCP, anti CD8a-FITC, anti CD25-APC antibodies (each $1 \mu \mathrm{g}$ ) for 45 minutes on ice under dark conditions. The cells were then stained with anti Foxp3PE antibody $(1 \mu \mathrm{g})$ using a Foxp3 staining buffer kit (eBioscience) for cell fixation and permeabilization. For Th17, cells in suspension were stained with anti CD4FITC (1 $\mu$ g, Biolegend) for 45 minutes on ice under dark conditions followed by intercellular staining with anti-IL

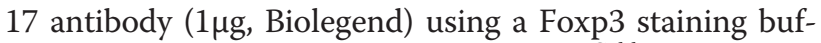
fer kit. The cells were analyzed on FACS ${ }^{\text {Calibur }}$.

\section{Allogenic mouse S-BMMSC transplantation into MRL/lpr mice}

Under general anesthesia, C3H/HeJ-derived BMMSCs, S-BMMSCs, L-NMMA pre-treated BMMSCs $(1 \mathrm{mM}$ for five days), or $\mathrm{CD} 34^{+} / \mathrm{CD} 3^{+}$double sorted cells $(0.1 \times$ $10^{6}$ cells/10 g body weight) were infused into MRL/lpr mice via the tail vein at 10 weeks of age $(n=6$ each group). In the control group, MRL/lpr mice received PBS $(n=5)$. All mice were sacrificed at two weeks post transplantation for further analysis. The protein concentration in urine was measured using a Bio-Rad Protein Assay (Bio-Rad, Hercules, CA, USA).

\section{Measurement of autoantibodies, albumin, soluble runt- related NF- $\kappa$ B ligand (sRANKL) and C-terminal telopeptides of type I collagen (CTX)}

Peripheral blood serum samples were collected from mice. Autoantibodies, sRANKL and CTX were analyzed by ELISA using commercially available kits (anti-dsDNA antibodies and ANA; alpha diagnostics, albumin and sRANKL; R\&D Systems, CTX; Nordic Bioscience Diagnostics, Herlev, Rigion Hovedstaden, Denmark) according to their manufactures' instructions. The results were averaged in each group. The intra-group differences were calculated between the mean values.

\section{Flow cytometric analysis of Tregs and Th17 cells}

To detect Tregs, peripheral blood mononuclear cells (PBMNCs) $\left(1 \times 10^{6}\right)$ were treated with PerCP-conjugated anti-CD4, FITC-conjugated anti-CD8a, APC-conjugated anti-CD 25 antibodies, and stained with R-PEconjugated anti-Foxp3 antibody using a Foxp3 staining buffer kit (eBioscience). To measure Th17 cells, PBMNCs $\left(1 \times 10^{6}\right)$ were incubated with PerCP-conjugated antiCD4, FITC-conjugated anti-CD8a, followed by treatment with R-PE-conjugated anti-IL-17 and APC-conjugated anti-IFN $\gamma$ antibodies using a Foxp3 staining buffer kit. The cells were then analyzed on FACS ${ }^{\text {Calibur }}$.

\section{Statistics}

Student's t-test was used to analyze statistical difference. $P$ values less than 0.05 were considered significant.

\section{Results}

A subset of BMMSCs lacks the ability to adhere to plastic culture dishes (S-BMMSCs) but attaches to extracellular cell matrix (ECM)-coated culture dishes

To determine whether a subset of BMMSCs remain in culture suspension, ANCs $\left(15 \times 10^{6}\right.$ cells $)$ from bone marrow were plated onto regular plastic culture dishes for two days and all non-attached cells were subsequently transplanted into immunocompromised mice subcutaneously using HA/TCP as a carrier. At eight weeks post-transplantation, newly formed bone was identified in the transplants by $\mathrm{H} \& \mathrm{E}$ staining (Figure $1 \mathrm{~A}$ ), suggesting that the BMMSC culture suspension may contain cells with a capacity to differentiate into bone forming cells. In vitro studies indicated that ECM produced by culture-expanded BMMSCs (BMMSC-ECM) could capture higher numbers of CFU-Fs when compared to plastic cultures [see 


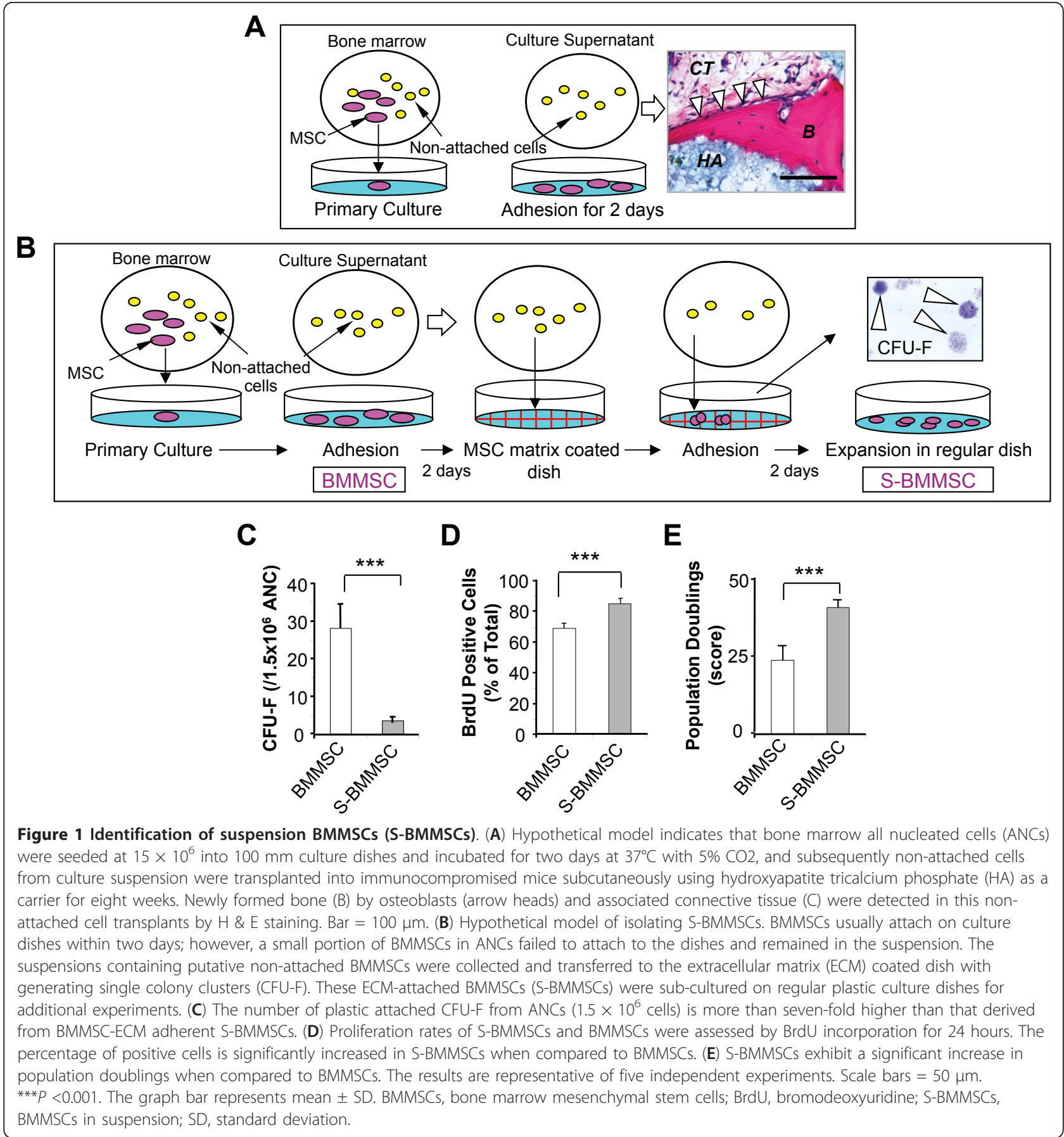

Additional file 1, Figure S1] [28]. Thus, we collected culture supernatant with floating cells at two days post CFU$F$ culture and re-loaded it onto BMMSC-ECM-coated dishes (Figure 1B). A subset of BMMSCs in the suspension (S-BMMSCs) was able to adhere to the BMMSC-ECM and form CFU-F (Figure 1B), at a lower incidence compared to the number of CFU-F generated from regular BMMSCs (Figure 1C). In order to characterize the stem cell properties of S-BMMSCs, we collected SSEA4-positive
S-BMMSCs and assessed their proliferation rate by BrdU incorporation. We found that S-BMMSCs had a significantly elevated BrdU uptake rate compared to regular BMMSCs (Figure 1D). In addition, we used a continuous cell culture assay to indicate that SSEA4-positive SBMMSCs acquired a significantly increased number of population doublings (Figure 1E). These data imply that SBMMSCs are distinct from regular BMMSCs in terms of attachment, proliferation, and self-renewal. 
To examine the multipotent differentiation potential, we showed that S-BMMSCs are analogous to BMMSCs in their expression of alkaline phosphatase (ALP), mineralized nodule accumulation under the osteogenic inductive cultures, and bone regeneration when transplanted into immunocompromised mice using HA/TCP as a carrier (Figures $2 \mathrm{~A}$ and 2B). Furthermore, we showed that S-BMMSCs were similar to regular BMMSCs in forming Oil red-O positive fat cells under adipogenic inductive conditions, which was associated with expression of the adipogenic genes, peroxisome proliferator-activated receptor gamma 2 (ppary 2$)$ and lipoprotein lipase ( $l p l$ ) (Figures $2 \mathrm{C}$ and 2D). Parallel studies showed a similar capacity between S-BMMSCs and regular BMMSCs to differentiate into chondrocytes under chondrogenic inductive conditions, associated with the expression of proteoglycan, trichrome positive collagen, and type II collagen (Figure 2E). Collectively, these data confirm that S-BMMSCs are a subset of BMMSCs.

\section{S-BMMSCs express CD34, but are distinct from hematopoietic stem cells}

By flow cytometric analysis, S-BMMSCs expressed mesenchymal stem cell markers at the same level as regular BMMSCs (Figure 3A). Interestingly, 23.4\% of S-BMMSCs expressed CD34, a hematopoietic stem cell (HSC) and endothelial cell marker, whereas $0.2 \%$ of BMMSCs expressed CD34 (Figure 3A). BMMSCs (21.4\%) and S-BMMSCs (31.2\%) expressed CD45, another hematopoietic marker, at passage 2 (Figure 3A). Both BMMSCs and S-BMMSCs were negative to CD11b antibody staining (data not shown), excluding the possibility that S-BMMSCs are derived from monocyte/macrophage lineage cells. Importantly, CD $34^{+}$S-BMMSCs co-expressed BMMSC-associated markers CD73 or Octamer-4 (Oct4),

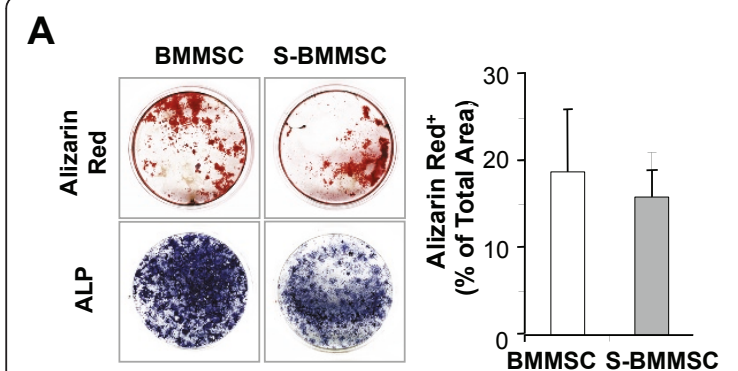

B

C
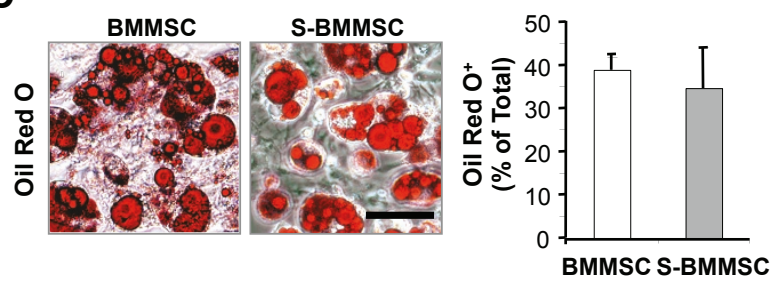
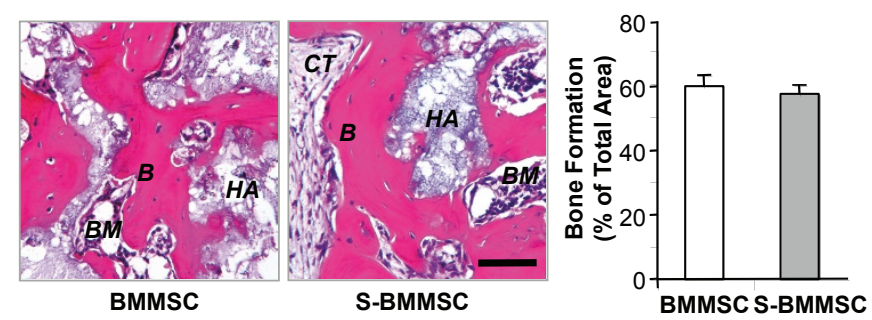

D

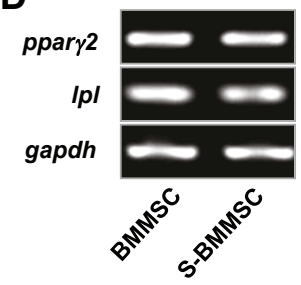

E

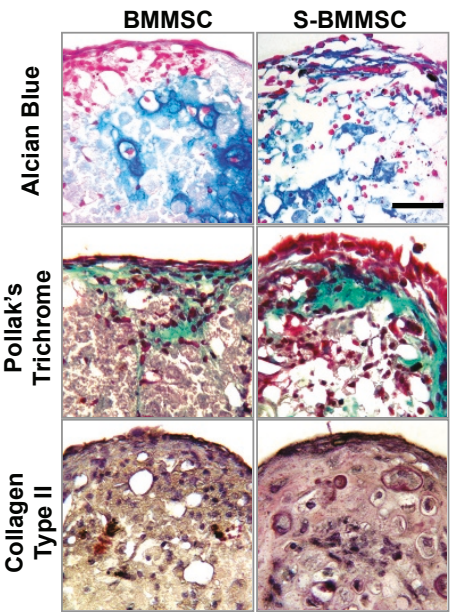

Figure 2 Multipotent differentiation of S-BMMSCs. (A) Alizarin Red S and alkaline phosphatase (ALP) staining showed that S-BMMSCS were similar to regular BMMSCs in osteogenic differentiation in vitro. (B) S-BMMSCs or regular BMMSCs $\left(4 \times 10^{6}\right.$ cells/transplant) were transplanted into immunocompromised mice using HA/TCP (HA) as a carrier for eight weeks. Bone formation was detected in S-BMMSC and BMMSC transplants, evidenced by $\mathrm{H}$ \& E staining. $\mathrm{HA}$, hydroxyapatite tricalcium phosphate; B, bone; $\mathrm{M}$, bone marrow; $\mathrm{CT}$, connective tissue. Bar = $50 \mu \mathrm{m}$. (C-D) S-BMMSCs are capable of forming Oil Red O positive cells (C) and expression of ppary2 and Ip/ mRNA as seen in regular BMMSCs (D). Glyceraldehyde 3-phosphate dehydrogenase (gapdh) was used as an internal control. The results are representative of five independent experiments. Scale bars $=100 \mu \mathrm{m}$. (E) Chondrogenic differentiation was assessed by Alcian blue staining for acidic sulfated mucosubstances, Pollak's Trichrome staining for collagen, and immunohistochemical staining for collagen type II. S-BMMSCs were able to differentiate into chondrocytes as observed in regular BMMSCs. Bar $=50 \mu \mathrm{m}$. The results are representative of three independent experiments. The graph bar represents mean $\pm \mathrm{SD}$. BMMSCs, bone marrow mesenchymal stem cells; S-BMMSCs, BMMSCs in suspension; SD, standard deviation. 


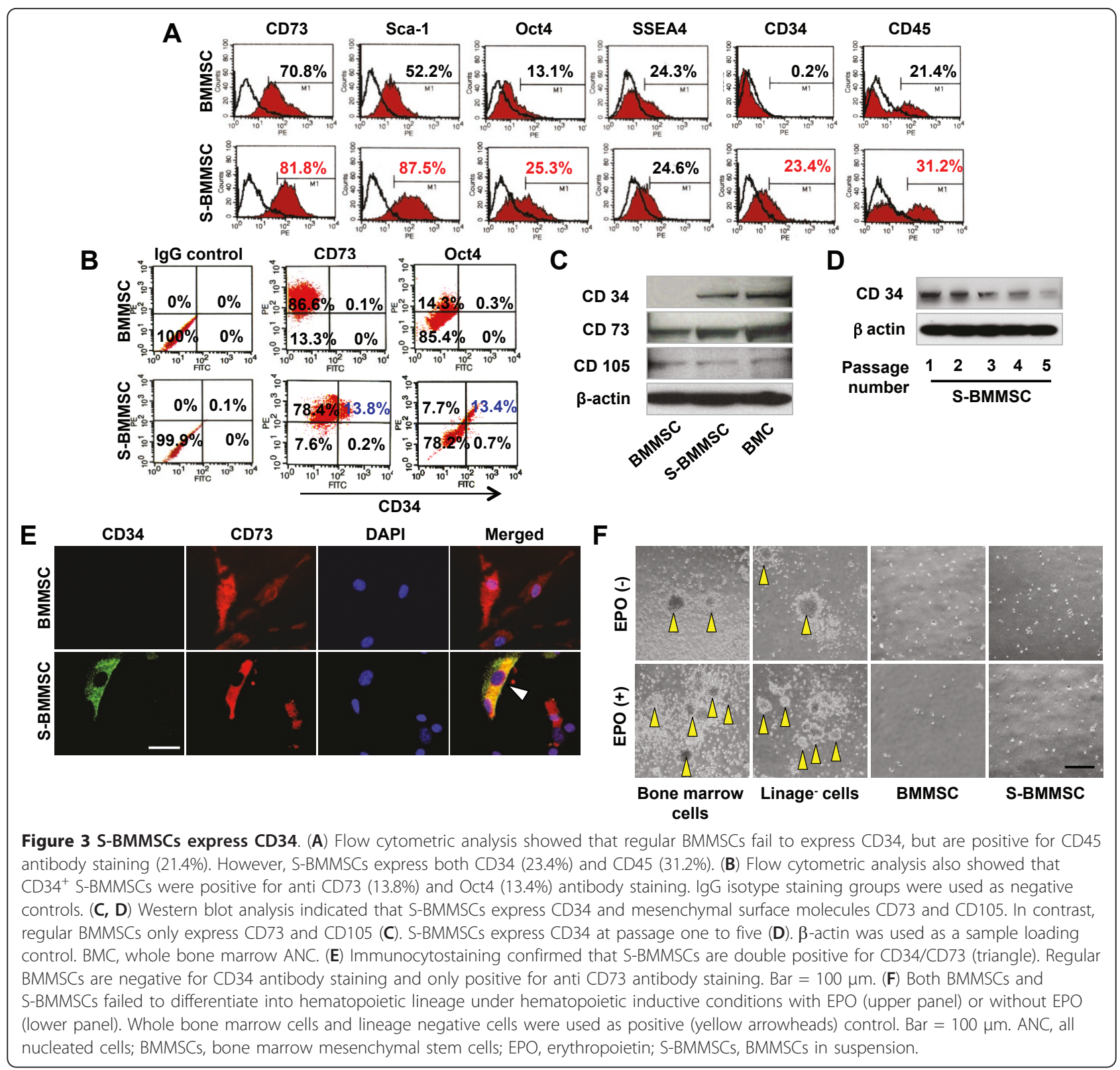

as evidenced by flow cytometric analysis (Figure 3B). Western blot analysis confirmed that S-BMMSCs expressed CD34, CD73, and CD105 (Figure 3C), and regular BMMSCs expressed CD73 and CD105 but lacked CD34 expression (Figure 3C). Whole bone marrow cells (BMC) were used as positive control. S-BMMSCs also showed a continued expression of CD34 from passage one to five; however, the expression levels appear reduced after passage three (Figure 3D). In order to further verify CD34 expression in S-BMMSCs, immunocytostaining analyses were performed to show co-expression of CD34 with mesenchymal markers CD73 (Figure 3E) in contrast to regular BMMSCs that were negative for anti-CD34 antibody staining (Figure 3E).
It is generally believed that CD34 expression is associated with HSCs and endothelial populations. HSCs can differentiate into all the blood cell lineages and rescue lethally irradiated subjects. Thus, we cultured S-BMMSCs and regular BMMSCs in hematopoietic differentiation medium and determined that these mesenchymal cells failed to differentiate into a hematopoietic cell lineage compare to bone marrow cells that formed myeloid and erythroid colony forming clusters (Figure 3F). In addition, CD45 CD34-BMMSCs showed an ability similar to that of S-BMMSCs in colony forming and expressing surface marker as MSC [see Additional file 1, Figure S2]. Furthermore, we infused S-BMMSCs systemically to rescue lethally irradiated 
mice and found that S-BMMSCs, but not regular BMMSCs, could extend the lifespan of lethally irradiated mice [see Additional file 1, Figure S3]. However, S-BMMSCs failed to rescue lethally irradiated mice, as shown in the whole bone marrow cell group [see Additional file 1, Figure S3]. These data provid further evidence that CD34 expression in S-BMMSCs is not due to HSC contamination.

\section{S-BMMSCs transplantation ameliorates multiple organ dysfunctions in MRL/lpr mice}

Since the immunomodulation property of MSCs is one of the essential factors for MSC characterization, allogenic S-BMMSC transplantation into MRL/lpr mice was performed (Figure 4A). Two weeks after transplantation, both S-BMMSCs and BMMSCs were capable of ameliorating SLE-induced glomerular basal membrane disorder (yellow arrow, Figure 4B) and reducing the urine protein level (Figure 4C). It appeared that S-BMMSCs were superior compared to BMMSCs in terms of reducing the overall urine protein levels (Figure 4C). As expected, MRL/lpr mice showed remarkably increased levels of autoantibodies, including anti-double strand DNA (dsDNA) IgG and IgM antibodies (Figures 4D and 4E) and anti-nuclear antibody (ANA; Figure 4F) in the peripheral blood serum. Although S-BMMSC and BMMSC infusion showed significantly decreased serum levels of anti-dsDNA IgG, IgM antibodies and ANA in peripheral blood (Figures 4D-F), S-BMMSCs showed a superior therapeutic effect in reducing anti-dsDNA IgG antibody and ANA levels when compared to BMMSCs (Figures 4D and 4F). Additionally, decreased serum albumin levels in MRL/lpr mice were recovered by S-BMMSC and BMMSC infusion (Figure 4G) but S-BMMSC treatment resulted in a more significant recovery than BMMSC treatment (Figure 4G). Next, flow cytometric analysis revealed that S-BMMSC showed more effectiveness in recovering the decreased level of $\mathrm{CD} 4^{+} \mathrm{CD} 25^{+} \mathrm{Foxp} 3^{+}$ Tregs and increased the number of $\mathrm{CD}^{+} \mathrm{IL} 17^{+} \mathrm{IFN} \gamma^{-} \mathrm{T}$ lymphocytes (Th17 cells) in peripheral blood when compared to BMMSCs (Figures 4H, 4I). In addition, highly passaged mouse S-BMMSCs failed to inhibit Th17 differentiation in vitro (data not shown) suggesting that mouse S-BMMSCs probably lose their immunomodulation property under long culture expansion.

Furthermore, we showed that S-BMMSCs were superior to BMMSCs in terms of reducing increased numbers of tartrate-resistant acid phosphatase (TRAP) positive osteoclasts in the distal femur epiphysis of MRL/lpr mice [see Additional file 1, Figure S4A], elevated serum levels of sRANKL, a critical factor for osteoclastogenesis [see Additional file 1, Figure S4B] and bone resorption marker CTX [see Additional file 1, Figure S4C]. These data suggest that S-BMMSCs exhibit a superior therapeutic effect for SLE disorders compared to regular BMMSCs.

\section{S-BMMSCs possess superior immunomodulatory functions via high nitric oxide (NO) production}

Recently, immunomodulatory properties were identified as an important stem cell characteristic of BMMSCs, leading to the utilization of systemic infused BMMSCs to treat a variety of immune diseases [19-21]. Here, we found that S-BMMSCs exhibited a significantly increased capacity for NO production compared to regular BMMSCs when treated with IFN $\gamma$ and IL-1 $\beta$ (Figure 5A). It is known that NO plays a critical role in BMMSCmediated immunosuppression [see Additional file 1, Figures S5A-F] [29]. Therefore, we assessed the functional role of high NO production in S-BMMSC-associated immunomodulatory properties. Spleen (SP) cells were activated by anti-CD3 and anti-CD28 antibodies for three days and then co-cultured with S-BMMSCs or regular BMMSCs in the presence of the general NOS inhibitor, L-NMMA or the iNOS inhibitor, $1400 \mathrm{~W}$, using a Transwell culture system. The efficacy of L-NMMA and 1400 $\mathrm{W}$ to inhibit NO production in BMMSCs was verified [see Additional file 1, Figures S6A and 6B]. Although both S-BMMSCs and regular BMMSCs were capable of inhibiting cell viability of activated SP cells, S-BMMSCs showed a marked inhibition of SP cell viability over that of regular BMMSCs (Figure 5B). Moreover, both BMMSCs and S-BMMSCs induced SP cell apoptosis (Figure 5C). However, S-BMMSCs showed an elevated capacity in inducing activated SP cell apoptosis compared to regular BMMSCs (Figure 5C). Interestingly, when LNMMA and $1400 \mathrm{~W}$ were added to the cultures, the number of apoptotic SP cells was significantly reduced in both S-BMMSC and regular BMMSC groups (Figure 5D and $5 \mathrm{E}$ ). These in vitro experimental data suggested that $\mathrm{NO}$ production is an essential factor for BMMSCmediated immunomodulation.

Since up-regulation of $\mathrm{CD} 4^{+} \mathrm{CD} 25^{+} \mathrm{Foxp}^{+}$Tregs is required for immunotolerance [30], we tested Tregs upregulation property of S-BMMSCs and BMMSCs in an in vitro co-culture system. When naïve- $\mathrm{T}$-cells were cocultured with S-BMMSCs or regular BMMSCs in the presence of IL- 2 and TGF- $\beta 1$, S-BMMSCs showed a significant up-regulation of Treg levels compared to regular BMMSCs (Figure 5F). Both L-NMMA and $1400 \mathrm{~W}$ were able to inhibit BMMSC- and S-BMMSC-induced up-regulation of Tregs, as shown by flow cytometric analysis (Figures 5G and 5H). Interestingly, the regulation effect on Tregs was more significant in the S-BMMSC group compared to the BMMSC group (Figure $5 \mathrm{G}$ and $5 \mathrm{H}$ ). Moreover, both BMMSCs and S-BMMSCs could inhibit differentiation of Th17 in vitro, with a more prominent 


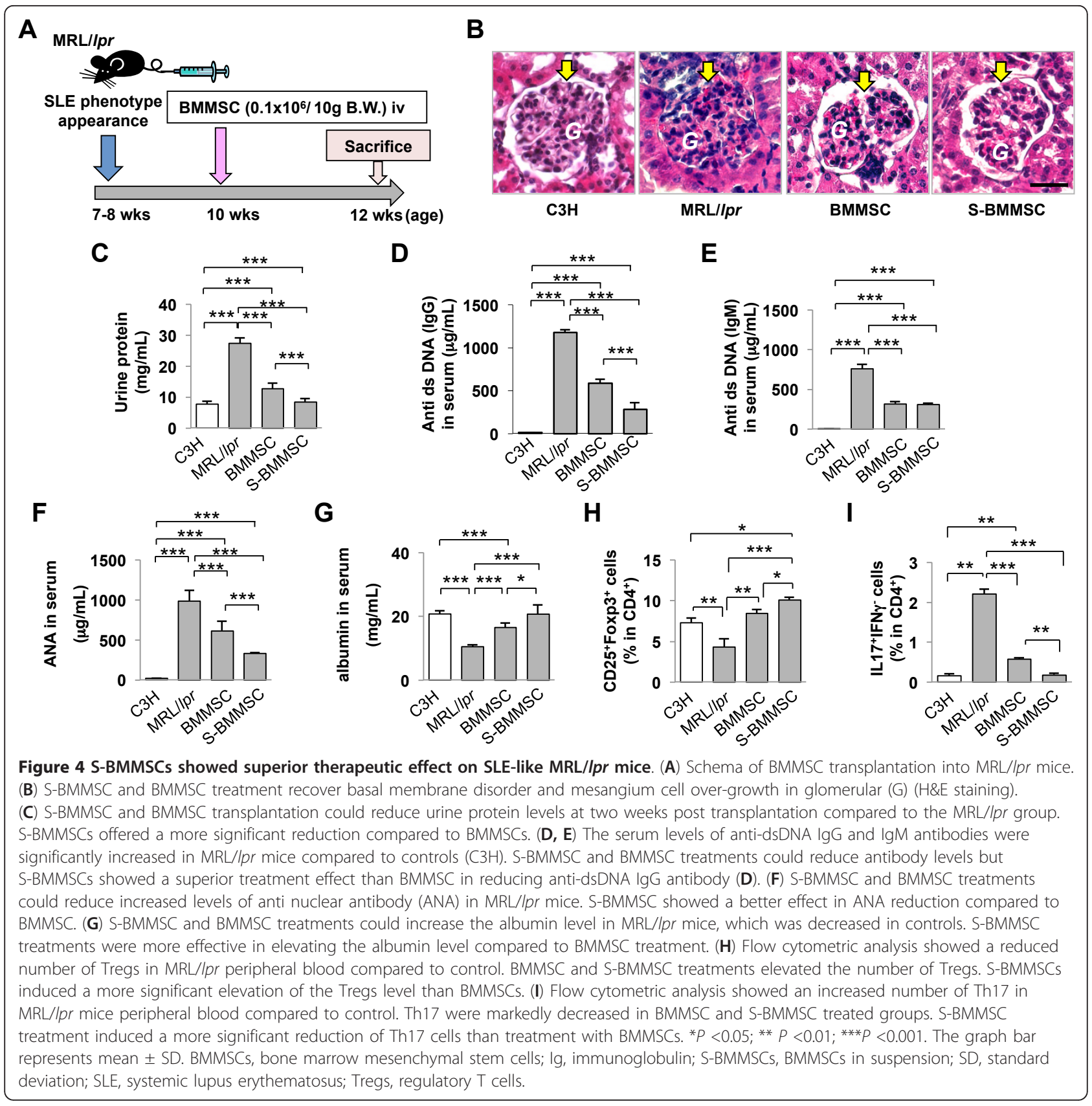

effect observed with S-BMMSC (Figure 5I). These inhibitions of Th17 differentiation were abolished by L-NMMA (Figure 5J) and $1400 \mathrm{~W}$ (Figure 5K). These data further verified the functional role of NO in S-BMMSC-induced immunomodulatory effect.

In order to identify whether there are functional endogenous S-BMMSCs, we used fluorescence activated cell sorting (FACS) to isolate CD34 and CD73 double-positive cells from bone marrow ANCs which resulted in the recovery of $3.77 \%$ double-positive cells [see Additional file 1, Figure S7A]. These CD34 and CD73 double-positive cells exhibited mesenchymal stem cell characteristics, including the capacity to form single colony clusters of fibroblast-like cells [see Additional file 1, Figure S7B], which could differentiate into osteogenic cells in vitro [see Additional file 1, Figure S7C]. These data indicated the feasibility of this approach to isolate S-BMMSC-like cells directly from bone marrow. We found that $\mathrm{CD} 34^{+} / \mathrm{CD} 3^{+}$BMMSCs were analogous to $\mathrm{S}$-BMMSCs in terms of having higher levels of NO production when compared to regular BMMSCs [see Additional file 1, Figure S7D] and reducing levels of urine 


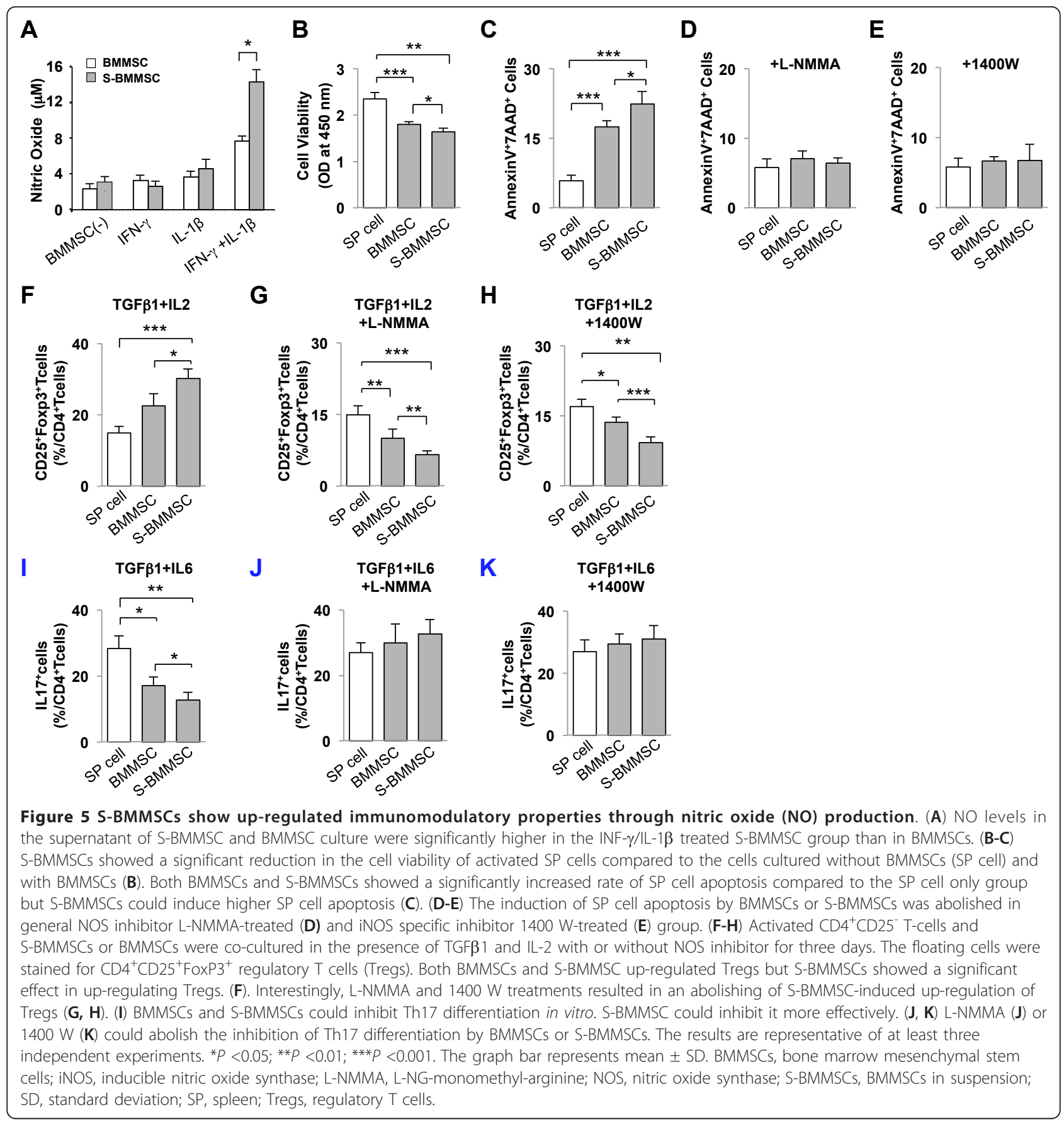

protein, serum anti-dsDNA IgG and IgM antibodies in MRL/lpr mice (data not shown). These data indicate that endogenous S-BMMSCs could be isolated from bone marrow using CD34 and CD73 antibodies double sorting.

Additionally, we used the same BMMSC-ECM isolation approach to reveal the existence of human SBMMSCs (hS-BMMSC) that possess stem cell properties including multipotent differentiation and self-renewal but lack expression of CD34 (data not shown). hSBMMSCs showed elevated NO and kynurenine production which indicate high indoleamine 2,3-dioxygenase (IDO) activity when compared to regular BMMSCs [see Additional file 1, Figures S8A-C]. Thus, when activated $\mathrm{T}$ cells were co-cultured with hS-BMMSCs, AnnexinV-7 aminoactinomycinD (7AAD) double positive apoptotic SP cells were significantly elevated compared to BMMSCs [see Additional file 1, Figure S8D]. 


\section{Discussion}

Adherent BMMSCs are able to proliferate and undergo osteogenic differentiation, providing the first evidence of CFU-F as precursors for osteoblastic lineage [25]. For over a few decades, the adherent CFU-F assay has been used as an effective approach to identify and select BMMSCs. In the current study, we showed that the adherent CFU-F assay collects the majority of clonogenic BMMSCs, but a subpopulation of BMMSCs is sustained in the culture suspension. This newly identified subpopulation of BMMSCs may be lost in the standard CFU-F assay for BMMSC isolation.

Due to the heterogeneity of the BMMSCs, there is no single, unique marker allowing for BMMSC isolation, rather an array of cell molecules are utilized to profile BMMSCs. It is widely accepted that BMMSCs express SH2 (CD105), SH3/SH4 (CD73), integrin $\beta_{1}$ (CD29), CD44, Thy-1 (CD90), CD71, vascular cell adhesion molecule-1 (CD106), activated leukocyte cell adhesion molecule (CD166), STRO-1, GD2, and melanoma cell adhesion molecule (CD146) [5,7-13,31,32]. Nevertheless, it is believed that BMMSCs lack expression of hematopoietic surface molecules including CD34, integrin $\alpha_{M}$ (CD11b) and CD14. However, recent studies have implied that mouse BMMSCs might express the hematopoietic surface molecules, CD45 [28] and CD34 [33]. To ensure purity of S-BMMSCs, we used immune FACS to collect SSEA4 ${ }^{+}$S-BMMSCs for proliferation and differentiation assays in this study. Interestingly, previous experimental evidence appeared to support a notion that HSCs are capable of differentiating into mesenchymal cells [34] and osteoblastic lineage in vivo [35]. Thus, it is critical to clarify whether BMMSCs express hematopoietic associated surface molecules.

In this study, we have identified a novel subset of SBMMSCs that failed to form adherent CFU-F in regular culture dishes, but were capable of adhering on mesenchymal stem cell-produced ECM and differentiating into osteoblasts, adipocytes and chondrocytes from both $\mathrm{C} 3 \mathrm{H} / \mathrm{HeJ}$ and $\mathrm{C} 57 \mathrm{BL} / 6 \mathrm{~J}$ mice. S-BMMSCs coexpressed the HSC marker CD34 with the MSC markers CD73 and Oct4, excluding the potential of HSC contamination. Furthermore, S-BMMSCs were found to be distinct from HSC because they lacked the ability to differentiate into hematopoietic cell lineages in vitro and failed to rescue lethally-irradiated mice. The mechanism that may contribute to the up-regulated immunomodulatory function was associated with high NO production in S-BMMSCs and a NO-driven high Tregs level [36]. NO is a gaseous biological mediator with important roles in affecting $\mathrm{T}$ cell function [37].

This is the reason that S-BMMSCs showed a superior therapeutic effect in treating SLE mice.
One successful approach is to isolate cells that express specific molecules on their cell surfaces using monoclonal antibodies and cell sorting technologies. Enriched populations of BMMSCs have been isolated from human bone marrow aspirates using a STRO-1 monoclonal antibody in conjunction with antibodies against VCAM-1/CD106 [32], CD146 [11], low affinity nerve growth factor receptor/CD271, PDGR-R, EGF-R and IGF-1-R [38], fibroblast cell marker/D7-Fib [39] and integrin alpha 1/CD49a [40]. A more recent study has also identified molecules co-expressed by a CD271 ${ }^{+}$ mesenchymal stem cell population including platelet derived growth factor receptor- $\beta$ (CD140b), human epidermal growth factor 2/ErbB2 (CD340) and frizzled-9 (CD349) [41]. Further cell separation based upon multiparameter FACS identified a population of proposed mouse mesenchymal precursors with the composite phenotype $\mathrm{Lin}^{-} \mathrm{CD} 45^{-} \mathrm{CD} 31^{-} \mathrm{Sca}-1^{+}$[42]. Another recent study also identified and characterized an alternate population of primitive mesenchymal cells derived from adult mouse bone marrow, based upon their expression of the SSEA-1 [43]. All approaches used for BMMSC purification and isolation will undergo ex vivo expansion to enrich cell numbers for tissue regeneration or systemic therapies by plastic adherent assay. In addition to identifying a novel sub-population of BMMSCs that possess enhanced immunomodulatory properties when compared to regular BMMSCs, we showed that CD34 ${ }^{+} / \mathrm{CD}{ }^{+}$BMMSCs could be isolated directly from whole bone marrow and that $\mathrm{CD} 34^{+} / \mathrm{CD}^{+} 3^{+}$BMMSCs are endogenous S-BMMSCs with higher NO production, and are superior in treating SLE-like mice when compared to regular BMMSCs.

Recently, non-adherent bone marrow cells (NABMCs) were identified $[44,45]$. The NA-BMSCs could be expanded in suspension and gave rise to multiple mesenchymal phenotypes, including osteoblasts, chondrocytes, and adipocytes in vitro, suggesting the presence of non-adherent BMMSCs in primary CFU-F cultures [45]. Although it has been reported that the NA-BMCs can rescue lethally-irradiated mouse recipients, our data indicated that S-BMMSCs only showed improved survival lifespan without a complete rescue of lethally-irradiated mice, compared to whole bone marrow transplantation. While the mechanism of SBMMSC-mediated lifespan extension in lethally-irradiated mice is unknown, it is possible that S-BMMSCs have a more active interplay with hematopoietic cells than regular BMMSCs. It has been reported that granulocyte colony stimulating factor might promote BMMSCs into the circulation in humans [46], suggesting that non-attached BMMSCs may exist in vivo for specific functional needs. Added evidence indicated that 
osteocalcin-positive cells in circulation were able to differentiate into osteoblastic cells when cultured in the presence of TGF $\beta$ [47]. However, it is unknown whether S-BMMSCs are associated with circulating mesenchymal stem cells initially identified in mice, and this is very rare in humans.

\section{Conclusions}

A new subset of BMMSCs (S-BMMSCs) which failed to adhere to culture dishes possesses similar stem cell properties as those seen in BMMSCs, including CFU-F, stem cell markers, osto-, adipo-, and chondro-genic differentiation. However, S-BMMSC showed distinct features including expression of CD34 and a superior immunomodulation property through high NO production. These findings suggest that it is feasible to improve immunotherapy by identifying new subset BMMSCs.

\section{Additional material}

Additional file 1: Figures S1 to S8 and Additional materials and
methods. Figure S1. ECM coated dish could capture a greater
number of CFU-F. CFU-f number in ECM coated dish compared to
regular dish. Figure S2. CD45 CD34-BMMSCs showed similar property
with S-BMMSCs. (A) CFU-f number. (B) Flow cytometric analysis. Figure
S3. S-BMMSCs extended survival rate of lethal dose of irradiated
mice. The life span of irradiated mice. Figure S4. Osteoclast activity in
S-BMMSC-treated MRL/Ipr mice. (A) Osteoclast number. (B) sRANKL
level. (C) CTX level. Figure S5. L-NMMA pre-treated BMMSC
transplantation failed to ameliorate disease phenotype of MRL/lpr
mice. (A) Anti dsDNA (IgG) level. (B) Anti dsDNA (IgM) level. (C) Urine
protein level. (D) Tregs level. (E) Th17 level. (F) Ratio between Tregs/Th17.
Figure S6. Inhibition of NO production in BMMSCs. (A) NO level with
inhibitors. (B) iNOS level by western blot. Figure S7. Endogenous S-
BMMSCs in mice bone marrow. (A) Cell sorting result. (B) CFU-f
number. (C) Osteogenic differentiation in vitro. (D) NO level. Figure S8.
Human bone marrow contains S-BMMSCs (hS-BMMSCs). (A) NO level.
(B) Kynurenine production. (C) Kynurenine production in co-culture
system. (D) T cell apoptosis induction by hS-BMMSCs. Additional
materials and methods describe about TRAP staining, Histomotry, Rescue
lethal dose irradiated mice, and Isolation of CD34 ${ }^{+}$CD73 ${ }^{+}$double positive
cells.

\section{Abbreviations}

7AAD: 7aminoactinomycineD; ALP: alkaline phosphatase; ANCs: all nucleated cells; BMMSCs: bone marrow mesenchymal stem cells; BrdU:

bromodeoxyuridine; CFU-F: colony forming unit fibroblastic; CTX: C-terminal telopeptides of type I collagen; DAPI: 4', 6-diamidino-2-phenylindole; (D) MEM: (Dulbecco's) modified Eagle's medium; ECM: extracellular cell matrix; ELISA: enzyme-linked immunosorbent assay; EPO: erythropoietin; FACS: fluorescence-activated cell sorting; FBS: fetal bovine serums; FITC: fluorescein isothiocyanate; H \& E: hematoxylin and eosin; HA/TCP: hydroxyapatite/ tricalcium phosphate; HSC: hematopoietic stem cell; IDO: indoleamine 2,3dioxygenase; IFNץ: interferon gamma; IgG: immunoglobulin G; IL-1ß: interleukin-1 beta; iNOS: inducible NOS; L-NMMA: L-NG-monomethylarginine; |pl: lipoprotein lipase; NF-kB: nuclear factor-kappa B; NOS: nitric oxide synthase; PBMNCs: peripheral blood mononuclear cells; PBS: phosphate-buffered saline; PE: phycoerythrin; PFA: paraformaldehyde; pparyz: peroxisome proliferator-activated receptor gamma 2; RT-PCR: reverse transcriptase polymerase chain reaction; S-BMMSC: BMMSCs in suspension; SLE: systemic lupus erythematosus; SP: spleen; SRANKL: soluble runt-related
NF-KB ligand; SSEA: stage-specific embryonic antigen; TGF 3 : transforming growth factor beta; Th17: T helper 17 cells; TRAP: tartrate-resistant acid phosphatase; Tregs: regulatory $\mathrm{T}$ cells.

\section{Acknowledgements}

We thank Dr. Tao Cai from NIH for discussions and critical reading of the manuscript. This work was supported by grants from the National Institute of Dental and Craniofacial Research, National Institutes of Health, Department of Health and Human Services (R01DE017449 and R01 DE019932 to S.S.)

\section{Author details}

${ }^{1}$ Center for Craniofacial Molecular Biology, University of Southern California, 2250 Alcazar Street, CSA 103, Los Angeles, CA 90033, USA. ²Department of Oral Rehabilitation and Regenerative Medicine, Okayama University Graduate School of Medicine, Dentistry, and Pharmaceutical Science, 2-5-1 Shikata-cho, Kita-ku, Okayama 700-8525, Japan. ${ }^{3}$ Department of Molecular Cell Biology and Oral Anatomy, Kyushu University Graduate School of Dental Science, Fukuoka 812-8582, Japan. ${ }^{4}$ Research and Development Center for Tissue Engineering, Fourth Military Medical University, Xi'an, Shanxi, China. ${ }^{5}$ Division of Research, Department of Comprehensive Dentistry, The University of Texas Health Science Center at San Antonio, 7703 Floyd Curl Drive, San Antonio, Texas 78229-3900, USA. ' Mesenchymal Stem Cell Group, Department of Haematology, Institute of Medical and Veterinary Science/ Hanson Institute, Adelaide 5000, South Australia, Australia.

\section{Authors' contributions}

$K A$ and $Y Y$ : contributions to conception and design of experiments, acquisition of data, analysis and interpretation of data. TY, CC, LT, and YJ: contributions to acquisition of data, analysis and interpretation of data. XC and SG: contributions to drafting the manuscript and revising critically. SS: contributions to conception and design, drafting the manuscript, and giving final approval of the version to be published. All authors have read and approved the manuscript for publication.

\section{Competing interests}

The authors declare that they have no competing interests.

Received: 25 July 2012 Revised: 11 September 2012

Accepted: 25 September 2012 Published: 19 October 2012

\section{References}

1. Friedenstein AJ, Chailakhyan RK, Latsinik NV, Panasyuk AF, Keiliss-Borok IV: Stromal cells responsible for transferring the microenvironment of the hemopoietic tissues. Cloning in vitro and retransplantation in vivo. Transplantation 1974, 17:331-340.

2. Prockop DJ: Marrow stromal cells as stem cells for nonhematopoietic tissues. Science 1997, 276:71-74

3. Gang EJ, Bosnakovski D, Figueiredo CA, Visser JW, Perlingeiro RC: SSEA-4 identifies mesenchymal stem cells from bone marrow. Blood 2007, 109:1743-1751.

4. Greco SJ, Liu K, Rameshwar P: Functional similarities among genes regulated by OCT4 in human mesenchymal and embryonic stem cells. Stem Cells 2007, 25:3143-3154.

5. Conget PA, Minguell JJ: Phenotypical and functional properties of human bone marrow mesenchymal progenitor cells. J Cell Physiol 1999, 181:67-73.

6. Covas DT, Panepucci RA, Fontes AM, Silva WA Jr, Orellana MD, Freitas MC, Neder L, Santos AR, Peres LC, Jamur MC, Zago MA: Multipotent mesenchymal stromal cells obtained from diverse human tissues share functional properties and gene-expression profiles with CD146+ perivascular cells and fibroblasts. Exp Hematol 2008, 36:642-654.

7. Galmiche MC, Koteliansky VE, Brière J, Hervé P, Charbord P: Stromal cells from human long-term marrow cultures are mesenchymal cells that differentiate following a vascular smooth muscle differentiation pathway. Blood 1993, 82:66-76.

8. Haynesworth SE, Baber MA, Caplan Al: Cell surface antigens on human marrow-derived mesenchymal cells are detected by monoclonal antibodies. Bone 1992, 13:69-80.

9. Martinez C, Hofmann TJ, Marino R, Dominici M, Horwitz EM: Human bone marrow mesenchymal stromal cells express the neural ganglioside GD2: 
a novel surface marker for the identification of MSCs. Blood 2007 109:4245-4248.

10. Sacchetti B, Funari A, Michienzi S, Di Cesare S, Piersanti S, Saggio I, Tagliafico E, Ferrari S, Robey PG, Riminucci M, Bianco P: Self-renewing osteoprogenitors in bone marrow sinusoids can organize a hematopoietic microenvironment. Cell 2007, 131:324-336.

11. Shi S, Gronthos S: Perivascular niche of postnatal mesenchymal stem cells in human bone marrow and dental pulp. J Bone Miner Res 2003, 18:696-704.

12. Shi S, Gronthos S, Chen S, Counter CM, Robey PG, Wang C-Y: Bone formation by human postnatal bone marrow stromal stem cells is enhanced by telomerase expression. Nat Biotechnol 2002, 20:587-591.

13. Sordi V, Malosio ML, Marchesi F, Mercalli A, Melzi R, Giordano T, Belmonte N, Ferrari G, Leone BE, Bertuzzi F, Zerbini G, Allavena P, Bonifacio $E$, Piemonti $L$ : Bone marrow mesenchymal stem cells express a restricted set of functionally active chemokine receptors capable of promoting migration to pancreatic islets. Blood 2005, 106:419-427.

14. Kwan MD, Slater BJ, Wan DC, Longaker MT: Cell-based therapies for skeletal regenerative medicine. Hum Mol Genet 2002, 17(R1):R93-98.

15. Panetta NJ, Gupta DM, Quarto N, Longaker MT: Mesenchymal cells for skeletal tissue engineering. Panminerva Med 2009, 51:25-41.

16. Liu Y, Wang L, Kikuiri T, Akiyama K, Chen CD, Xu XT, Yang RL, Chen WJ, Wang SL, Shi S: Mesenchymal stem cell-based tissue regeneration is governed by recipient T lymphocytes via IFN-Gamma and TNF-Alpha. Nat Medicine 2011, 17:1594-1601.

17. Nauta AJ, Fibbe WE: Immunomodulatory properties of mesenchymal stromal cells. Blood 2007, 110:3499-3506.

18. Uccelli A, Pistoia V, Moretta L: Mesenchymal stem cells: a new strategy for immunosuppression? Trends Immunol 2007, 28:219-226.

19. Uccelli A, Moretta L, Pistoia V: Mesenchymal stem cells in health and disease. Nat Rev Immunol 2008, 8:726-736.

20. Aggarwal S, Pittenger MF: Human mesenchymal stem cells modulate allogeneic immune cell responses. Blood 2005, 105:1815-1822.

21. Bernardo ME, Locatelli F, Fibbe WE: Mesenchymal stromal cells. Ann N Y Acad Sci 2009, 1176:101-117.

22. Le Blanc K, Rasmusson I, Sundberg B, Götherström C, Hassan M, Uzunel M, Ringdén $\mathrm{O}$ : Treatment of severe acute graft-versus-host disease with third party haploidentical mesenchymal stem cells. Lancet 2004, 363:1439-1441

23. Chen $X$, Armstrong MA, Li G: Mesenchymal stem cells in immunoregulation. Immunol Cell Biol 2006, 84:413-421.

24. Sun L, Akiyama K, Zhang H, Yamaza T, Hou Y, Zhao S, Xu T, Le A, Shi S: Mesenchymal stem cell transplantation reverses multi-organ dysfunction in systemic lupus erythematosus mice and humans. Stem Cells 2009, 27:1421-1432.

25. Friedenstein AJ: Stromal mechanisms of bone marrow: cloning in vitro and retransplantation in vivo. Haematol Blood Transfus 1980, 25:19-29.

26. Clarke E, McCann SR: Age dependent in vitro stromal growth. Bone Marrow Transplant 1989, 4:596-597.

27. Friedenstein AJ, Chailakhjan RK, Lalykina KS: The development of fibroblast colonies in monolayer cultures of guinea-pig bone marrow and spleen cells. Cell Tissue Kinet 1970, 3:393-403.

28. Chen XD, Dusevich V, Feng JQ, Manolagas SC, Jilka RL: Extracellular matrix made by bone marrow cells facilitates expansion of marrow-derived mesenchymal progenitor cells and prevents their differentiation into osteoblasts. J Bone Miner Res 2007, 22:1943-1956.

29. Ren G, Zhang L, Zhao X, Xu G, Zhang Y, Roberts Al, Zhao RC, Shi Y: Mesenchymal stem cell-mediated immunosuppression occurs via concerted action of chemokines and nitric oxide. Cell Stem Cell 2008, 2:141-150.

30. Perruche S, Zhang P, Liu Y, Saas P, Bluestone JA, Chen W: CD3-specific antibody-induced immune tolerance involves transforming growth factor-beta from phagocytes digesting apoptotic T cells. Nat Med 2008, 5:528-535.

31. Gronthos S, Zannettino AC, Hay SJ, Shi S, Graves SE, Kortesidis A, Simmons PJ: Molecular and cellular characterisation of highly purified stromal stem cells derived from human bone marrow. J Cell Sci 2003, 116:1827-1835.

32. Simmons PJ, Torok-Storb B: Identification of stromal cell precursors in human bone marrow by a novel monoclonal antibody, STRO-1. Blood 1991, 78:55-62.
33. Copland I, Sharma K, Lejeune L, Eliopoulos N, Stewart D, Liu P, Lachapelle K, Galipeau J: CD34 expression on murine marrow-derived mesenchymal stromal cells: impact on neovascularization. Exp Hematol 2008, 36:93-103.

34. Ogawa M, Larue AC, Watson PM, Watson DK: Hematopoietic stem cell origin of mesenchymal cells: opportunity for novel therapeutic approaches. Int J Hematol 2012, 91:353-359.

35. Olmsted-Davis EA, Gugala Z, Camargo F, Gannon FH, Jackson K, Kienstra KA Shine HD, Lindsey RW, Hirschi KK, Goodell MA, Brenner MK, Davis AR: Primitive adult hematopoietic stem cells can function as osteoblast precursors. Proc Natl Acad Sci USA 2003, 100:15877-15882.

36. Niedbala W, Cai B, Liu H, Pitman N, Chang L, Liew FY: Nitric oxide induces CD4+CD25+ Foxp3 regulatory T cells from CD4+CD25 T cells via p53, LL2, and OX40. Proc Natl Acad Sci USA 2007, 104:15478-15483.

37. Sato K, Ozaki K, Oh I, Meguro A, Hatanaka K, Nagai T, Muroi K, Ozawa K: Nitric oxide plays a critical role in suppression of T-cell proliferation by mesenchymal stem cells. Blood 2007, 109:228-234.

38. Gronthos S, Simmons PJ: The growth factor requirements of STRO-1positive human bone marrow stromal precursors under serum-deprived conditions in vitro. Blood 1995, 85:924-940.

39. Jones EA, Kinsey SE, English A, Jones RA, Straszynski L, Meredith DM, Markham AF, Jack A, Emery P, McGonagle D: Isolation and characterization of bone marrow multipotential mesenchymal progenitor cells. Arthritis Rheum 2002, 46:3349-3360.

40. Gronthos S, Simmons PJ, Graves SE, Robey PG: Integrin-mediated interactions between human bone marrow stromal precursor cells and the extracellular matrix. Bone 2001, 28:174-181.

41. Bühring HJ, Battula VL, Treml S, Schewe B, Kanz L, Vogel W: Novel markers for the prospective isolation of human MSC. Ann N Y Acad Sci 2007, 1106:262-271

42. Short B, Brouard N, Occhiodoro-Scott T, Ramakrishnan A, Simmons PJ: Mesenchymal stem cells. Arch Med Res 2003, 34:565-571.

43. Anjos-Afonso F, Bonnet D: Nonhematopoietic/endothelial SSEA- $1^{+}$cells define the most primitive progenitors in the adult murine bone marrow mesenchymal compartment. Blood 2007, 109:1298-1306.

44. Włodarski KH, Galus R, Włodarski P: Non-adherent bone marrow cells are a rich source of cells forming bone in vivo. Folia Biol (Praha) 2004, 50:167-173.

45. Zhang ZL, Tong J, Lu RN, Scutt AM, Goltzman D, Miao DS: Therapeutic potential of non-adherent BM-derived mesenchymal stem cells in tissue regeneration. Bone Marrow Transplant 2009, 43:69-81.

46. Lund TC, Tolar J, Orchard PJ: Granulocyte colony-stimulating factor mobilized CFU-F can be found in the peripheral blood but have limited expansion potential. Haematologica 2008, 93:908-912.

47. Eghbali-Fatourechi G, Lamsam J, Fraser D, Nagel D, Riggs BL, Khosla S: Circulating osteoblast-lineage cells in humans. N Engl J Med 2005, 352:1959-1966.

doi:10.1186/scrt131

Cite this article as: Akiyama et al:: Characterization of bone marrow derived mesenchymal stem cells in suspension. Stem Cell Research \& Therapy 2012 3:40.

\section{Submit your next manuscript to BioMed Central and take full advantage of:}

- Convenient online submission

- Thorough peer review

- No space constraints or color figure charges

- Immediate publication on acceptance

- Inclusion in PubMed, CAS, Scopus and Google Scholar

- Research which is freely available for redistribution

Submit your manuscript at www.biomedcentral.com/submit
C Biomed Central 